\title{
Study of Temporal Thermal Response of Microfiber Bragg Grating
}

\author{
Changrui LIAO ${ }^{1,2^{*}}$, Tianhang YANG ${ }^{1,2}$, and Jinli HAN ${ }^{1,2}$ \\ ${ }^{1}$ Shenzhen Key Laboratory of Photonic Devices and Sensing Systems for Internet of Things, College of Physics and \\ Optoelectronic Engineering, Shenzhen University, Shenzhen 518060, China \\ ${ }^{2}$ Guangdong and Hong Kong Joint Research Centre for Optical Fibre Sensors, Shenzhen University, Shenzhen 518060, \\ China \\ *Corresponding author: Changrui LIAO_ E-mail: cliao@szu.edu.cn
}

\begin{abstract}
Fiber Bragg grating has been successfully fabricated in the silica microfiber by the use of femtosecond laser point-by-point inscription. Temporal thermal response of the fabricated silica microfiber Bragg grating has been measured by the use of the $\mathrm{CO}_{2}$ laser thermal excitation method, and the result shows that the time constant of the microfiber Bragg grating is reduced by an order of magnitude compared with the traditional single-mode fiber Bragg grating and the measured time constant is $\sim 21 \mathrm{~ms}$.
\end{abstract}

Keywords: Fiber optics sensors; fiber optics and optical communications; fiber optics components

Citation: Changrui LIAO, Tianhang YANG, and Jinli HAN, "Study of Temporal Thermal Response of Microfiber Bragg Grating," Photonic Sensors, 2021, 11(4): 387-391.

\section{Introduction}

Temperature is one of the principal parameters in thermodynamics and temperature measurement, which plays an important role in industry, medicine, scientific research, and people's daily life [1-3]. Over the past few decades, different kinds of thermometers, such as glass liquid thermometer, thermocouple, and resistance thermometer, have been widely used. In the case of extreme environment such as corrosive, strong electromagnetic, flammable, and explosive, most of the traditional thermometers cannot meet the practical needs. Since silica fibers are good at high temperature stability, corrosion resistant, and anti-electromagnetic interference, silica-fiber based temperature sensors have attracted more and more attention recently [4-8]. Temporal thermal response of the sensing element is especially important for some rapidly changing and dynamic systems such as internal combustion engines. Liao et al. [9] have systemically studied temperature response of the femtosecond laser (FS) fabricated fiber Bragg gratings (FBGs) and found the temporal thermal response is dependent on the cross-sectional dimension of the fiber. Temporal thermal response has a linear relationship with the diameter of the fiber.

\section{Experimental details}

In this work, the silica microfiber is produced by the use of the flame brushing method $[10,11]$ in the fiber tapering system built in our lab. The single mode fiber (SMF) is fixed between two co-axial 
translation stages with fiber holders. The hydrogen flame with a diameter of $\sim 10 \mathrm{~mm}$ is mounted above the fiber in the middle of the two translation stages. The temperature of hydrogen flame is controlled by adjusting the flow rate of $\mathrm{H}_{2}$. The two translation stages with the same translation direction and different speeds of reciprocating motion are used to taper the fiber. Among them, the initial distance between the two stages is set as $15 \mathrm{~mm}$, the speeds of the two translation stages are set as $4.5 \mathrm{~mm} / \mathrm{s}$ and $5 \mathrm{~mm} / \mathrm{s}$, respectively, the time of the single movement is set as $3 \mathrm{~s}$, and the number of reciprocations is set as 25 . The microfibers with the diameter of $\sim 10 \mu \mathrm{m}$ and the uniform length of $\sim 40 \mathrm{~mm}$ have been prepared for FBG inscription [12].

Figure 1 shows the schematic diagram of the FBG fabricated in the silica microfiber by the use of the FS point-by-point inscription method. FS laser (Spectra-Physics, Solstice) producing $120 \mathrm{fs}$ pulses at $800 \mathrm{~nm}$ and a repetition rate of $1 \mathrm{kHz}$ is focused into the microfiber by the use of an oil-immersion objective with a numerical aperture (NA) value of 1.25. Figure 2 shows the schematic diagram of the FS laser micromachining system. The microfiber is mounted on a 3-dimensional (3D) translation stage (NEWPORT, XMS50/XMS50/GTS30V) to control the translation speed along the $X$ axis (fiber axis) and adjust the position of the microfiber along the $Y$ and $Z$ axes. FS laser energy is attenuated by rotating a $\lambda / 2$ plate followed by a polarizer $[13,14]$. The high-speed shutter is used to precisely control laser fabrication time. The charge coupled device (CCD) camera mounted top is used to monitor the fabrication process.

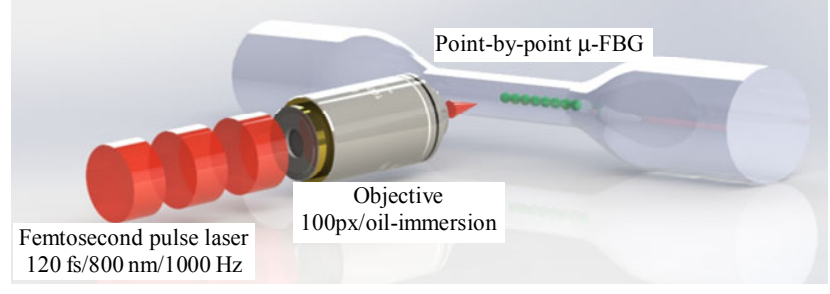

Fig. 1 Schematic diagram of the FBG fabricated in the silica microfiber by the use of the FS point-by-point inscription method.

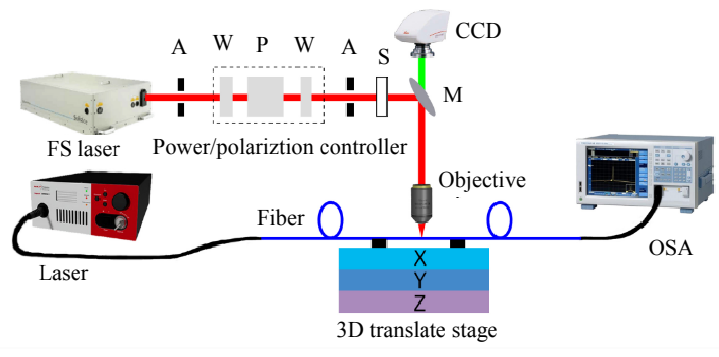

Fig. 2 Schematic diagram of the FS laser micromachining system, where $\mathrm{A}$ is an aperture; $\mathrm{W}$ is a $\lambda / 2$ plate; $\mathrm{P}$ is a polarizer; $\mathrm{S}$ is a shutter; $\mathrm{M}$ is a dichroic mirror; OSA is an optical spectrum analyzer.

To fabricate the $\mu$-FBG, the microfiber is firstly placed at the focal point of the FS laser beam and then moved with a designated speed along the $X$ axis. Each laser pulse produces one grating segment in the microfiber. To avoid the cylindrical lens effect from the fiber surface, we choose paraffin oil for the objective with the refractive index similar with silica [15]. In the experiment, the $\mu$-FBG with a grating period of $1.071 \mu \mathrm{m}$ and a uniform length of $\sim 4 \mathrm{~mm}$ has been fabricated in the microfiber with a diameter of $10 \mu \mathrm{m}$. The fabrication process takes $\sim 4 \mathrm{~s}$ and there is no material damage for the microfiber. Figure 3 shows the optical microscope image of the fabricated $\mu$-FBG, where the black dashed lines denote the boundary of the microfiber and the red dashed lines denote the Bragg grating with a grating period of $1.071 \mu \mathrm{m}$.

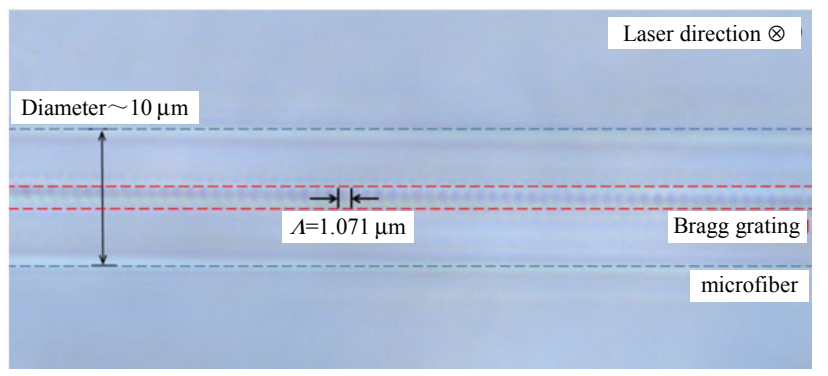

Fig. 3 Optical microscopic image of the $10 \mu \mathrm{m}$ FBG with the grating period of $1.071 \mu \mathrm{m}$.

The $10 \mu \mathrm{m}$ microfiber is a multimode fiber in C-band. Figure 4 shows the reflection and transmission spectra of the $10 \mu \mathrm{m}$ FBG, where more than one Bragg resonance can be observed and it can be explained by different-order reflection modes coupled from the core mode $[16,17]$. Not all the 
reflection peaks corresponding to each transmission dip can be observed in the reflection spectrum due to some high-order reflection modes, blocked by the adjacent SMF.

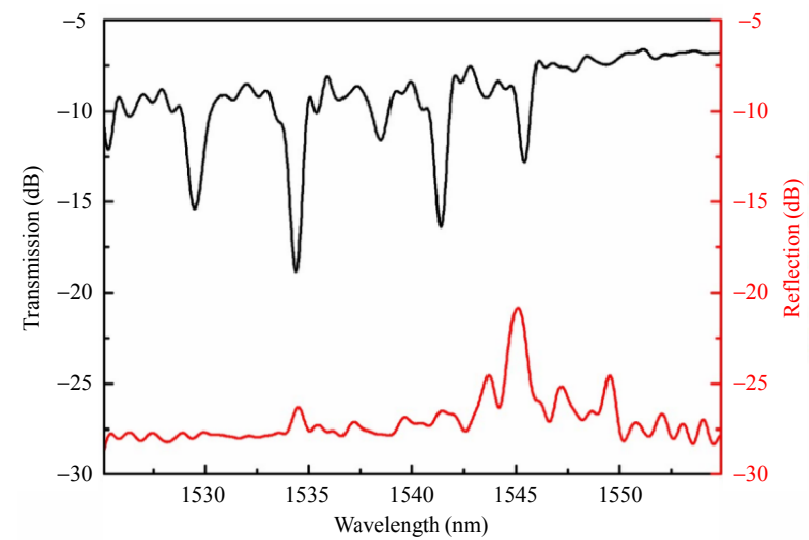

Fig. 4 Reflection and transmission spectra of the $10 \mu \mathrm{m}$ FBG.

Figure 5 shows the measurement setup of temporal thermal response of the $\mu$-FBG. $\mathrm{A} \mathrm{CO}_{2}$ laser with the wavelength of $10.6 \mu \mathrm{m}$ is used as the heat source since the silica fiber exhibits a high absorption efficiency at this wavelength. The $\mathrm{CO}_{2}$ laser beam is modulated by the use of an optical shutter (THORLABS, SC10) to warm or cool the $\mu$-fiber, operating as a chopper. The laser beam passes through a convex lens to broaden the beam diameter to irradiate the $\mu$-FBG uniformly. A tunable laser combining with a photodetector and an oscilloscope are used to record the dynamic change of the spectral signal.

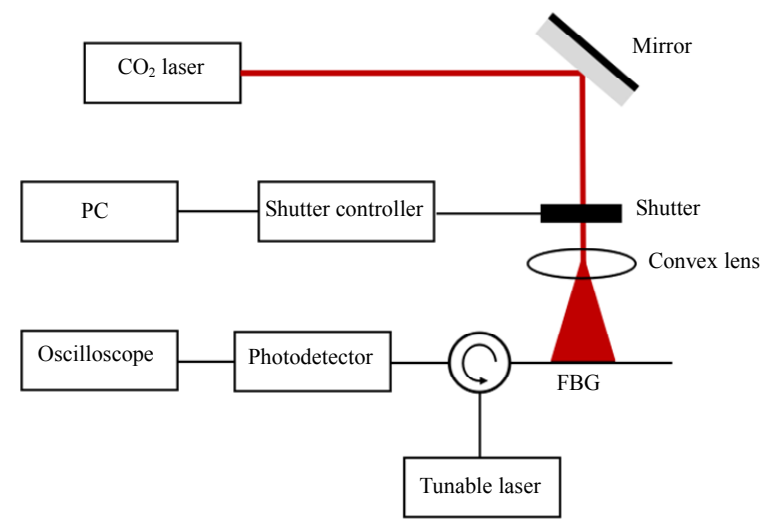

Fig. 5 Schematic diagram of the measurement setup of temporal thermal response of the $\mu$-FBG.

Figure 6(a) shows that the $\mu$-FBG is warmed by $\mathrm{CO}_{2}$ laser irradiation, the Bragg reflection peak will be shifted towards the longer wavelength, and as the thermal excitation is stopped, the reflection peak will be shifted back to the initial state quickly. To record the fast signal change with the temperature, it is necessary to use a fast demodulation system. To do so, the wavelength of tunable laser is set to be the left edge of the full width at half maximum (FWHM) of the fundamental-mode reflection peak, which is measured to be $\sim 0.95 \mathrm{~nm}$. As long as the $\mu$-FBG's spectrum is shifted to the longer wavelength by warming with $\mathrm{CO}_{2}$ laser, the tunable laser signal can be filtered along the rising edge of the $\mu$-FBG, resulting in a change in the photodetector output power. This signal is easily recorded on the oscilloscope, as shown in Fig. 6(b).

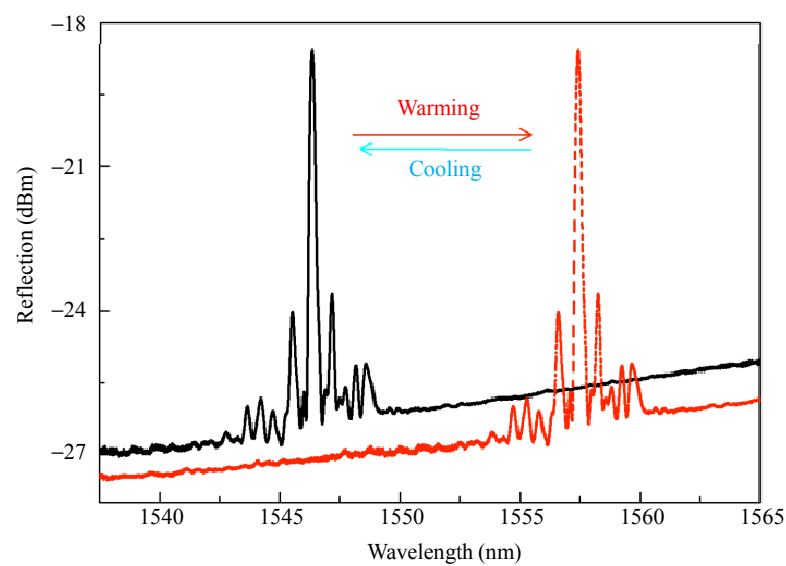

(a)

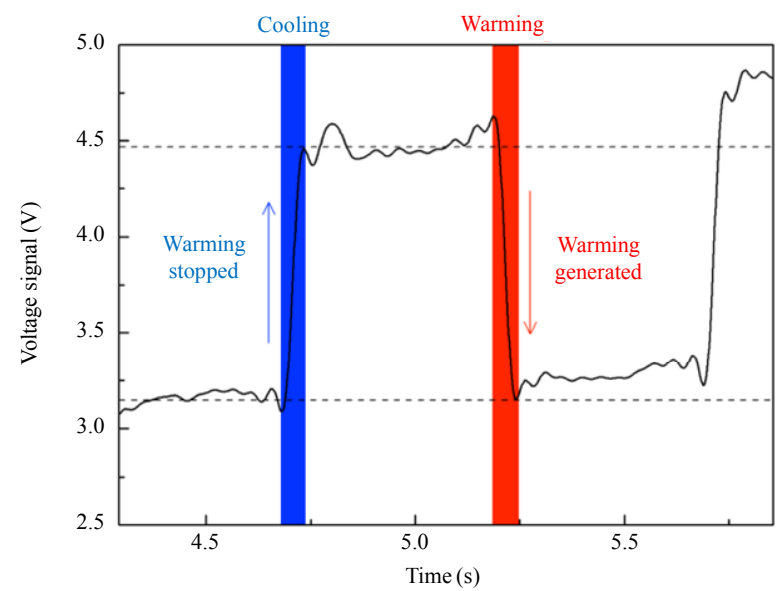

(b)

Fig. 6 Spectral response of the microfiber with thermal excitation: (a) reflection spectral evolution of the $\mu$-FBG with $\mathrm{CO}_{2}$ laser thermal excitation ON (warming) and OFF (cooling) and (b) voltage signal variation in the oscilloscope with periodical $\mathrm{CO}_{2}$ laser thermal excitation. 
Time constant is a feature of lumped system analysis (lumped capacity analysis method) for thermal systems, which is used when objects are cool or warm uniformly under the influence of convective cooling or warming. Theoretically, the time constant represents the elapsed time required for the system response to decay to zero if the system continues to decay at the initial rate, because of the progressive change in the rate of decay, and the response will eventually decrease in value to $1 / \mathrm{e} \approx 36.8 \%$ at the time. In an increasing system, the time constant is the time for the system's step response to reach $1-1 / \mathrm{e} \approx 63.2 \%$ of its final value.

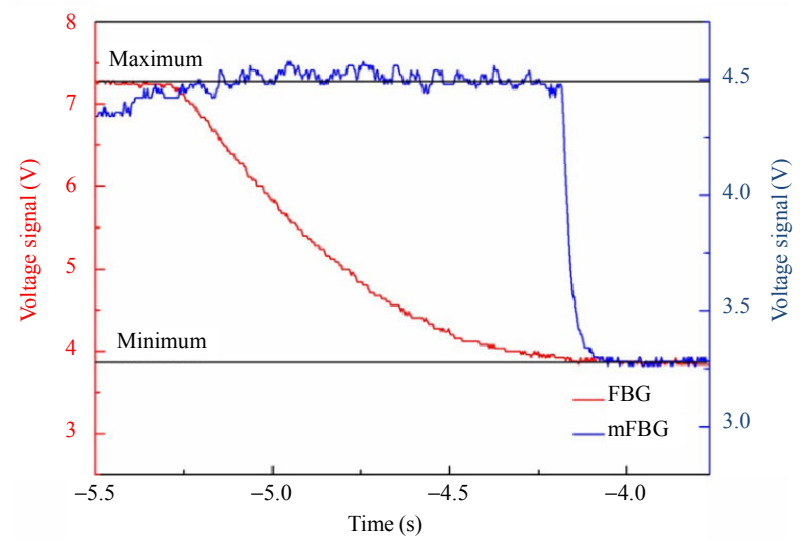

(a)
Figure 7 compares the time constants between the $\mu$-FBG and conventional FBG. A time period of $24 \mathrm{~s}(-12 \mathrm{~s}-+12 \mathrm{~s})$ is recorded in the oscilloscope. The time intervals of shutter being opened and closed are set to $3 \mathrm{~s}$. We calculate the time constants of four transient changes over $24 \mathrm{~s}$ and weight the data on average. The time constants in warming/cooling processes are measured to be $252 \mathrm{~ms} / 272 \mathrm{~ms}$ for the conventional FBG and $21 \mathrm{~ms} / 21 \mathrm{~ms}$ for the $\mu$-FBG. Obviously, the time constant of the $\mu$-FBG is smaller by an order of magnitude than that of the conventional FBG.

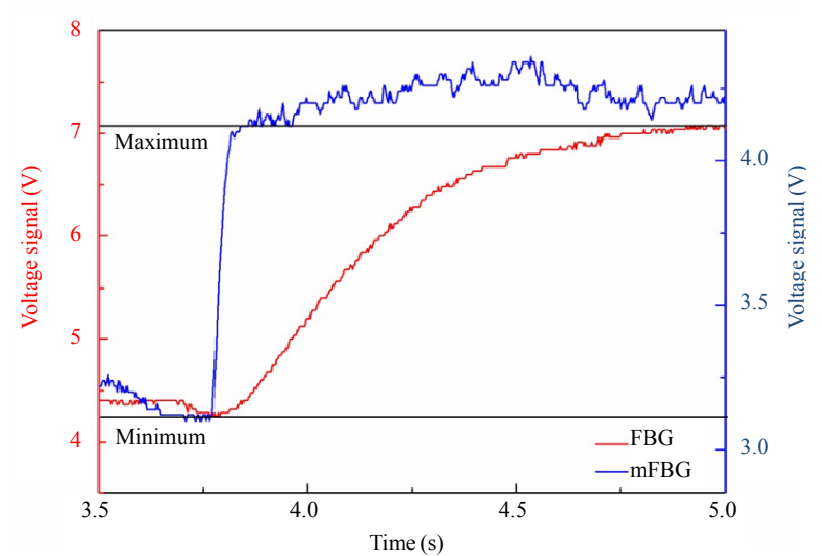

(b)

Fig. 7 Comparison of the time constant between the $\mu$-FBG and conventional FBG: (a) warming curves as a function of time of the $\mu$-FBG and conventional FBG and (b) cooling curves as a function of time of the $\mu$-FBG and conventional FBG.

The heat transfer between the $\mu$-FBG and the ambient at a given time is proportional to the temperature difference between the $\mu$-FBG and the ambient. Hence, warming and cooling of the $\mu$-FBG can be described by the lumped system equation $[18,19]$ :

$$
\begin{gathered}
\frac{\mathrm{d} T(t)}{\mathrm{d} t}=-\frac{1}{\tau}\left[T(t)-T_{\alpha}\right]+\Xi(t) \\
\tau=\frac{c_{p} \rho V_{f}}{A_{f} h} \\
\Xi(t)=\frac{q(t)}{c_{p} \rho}
\end{gathered}
$$

where $T(t)$ is the $\mu$-FBG temperature as a function of time; $T_{\alpha}$ is the temperature of ambient air $(295 \mathrm{~K})$; $A_{f}$ is the surface area of the fiber; $V_{f}$ is the volume of the fiber; $\rho$ is the density of the fiber $\left(2200 \mathrm{~kg} \cdot \mathrm{m}^{-3}\right)$; $c_{p}$ is the specific heat of the fiber $\left(837 \mathrm{~J} \cdot \mathrm{kg}^{-1} \cdot \mathrm{K}^{-1}\right)$; $h$ is the convection coefficient; $q(t)$ is the heat generation rate per unit volume; $\tau$ denotes the relaxation time; $\Xi(t)$ is periodic heating of the $\mathrm{CO}_{2}$ laser beam [20]. From (1), the time constants of the conventional FBG and $10 \mu \mathrm{m}$ FBG are calculated to be $250 \mathrm{~ms}$ and $20 \mathrm{~ms}$, which are in good agreement with the experimental results.

\section{Conclusions}

In conclusion, the $\mu$-FBG has been fabricated by the use of femtosecond laser point-by-point inscription. Due to the multimode operation of the $\mu$-FBG, multiple Bragg resonances are observed in the transmission spectrum. A fast-thermal excitation 
system is built to measure the time constant of thermal response and the time constant of the $\mu$-FBG with a diameter of $10 \mu \mathrm{m}$ measured to be $21 \mathrm{~ms}$, which is faster by an order of magnitude than that of the conventional FBGs. Such a $\mu$-FBG may be found potential sensing applications in some rapidly changing and dynamic systems.

Open Access This article is distributed under the terms of the Creative Commons Attribution 4.0 International License (http://creativecommons.org/licenses/by/4.0/), which permits unrestricted use, distribution, and reproduction in any medium, provided you give appropriate credit to the original author(s) and the source, provide a link to the Creative Commons license, and indicate if changes were made.

\section{References}

[1] K. O. Hill and G. Meltz, "Fiber Bragg grating technology fundamentals and overview," Journal of Lightwave Technology, 1997, 15(8): 1263-1276.

[2] Y. J. Rao, "In-fibre bragg grating sensors," Measurement Science and Technology, 1997, 8(4): 355-375.

[3] A. D. Kersey, M. A. Davis, H. J. Patrick, M. LeBlanc, K. P. Koo, C. G. Askins, et al., "Fiber grating sensors," Journal of Lightwave Technology, 1997, 15(8): 1442-1463.

[4] J. P. Dakin, D. J. Pratt, G. W. Bibby, and J. N. Ross, "Distributed optical fibre Raman temperature sensor using a semiconductor light source and detector," Electronics Letters, 1985, 21(13): 569-570.

[5] E. Li, X. Wang, and C. Zhang, "Fiber-optic temperature sensor based on interference of selective higher-order modes," Applied Physics Letters, 2006, 89(9): 091119.

[6] R. R. Dils, "High-temperature optical fiber thermometer," Journal of Applied Physics, 1983, 54(3): 1198-1201.

[7] C. Wang, J. He, J. C. Zhang, C. R. Liao, Y. Wang, W. Jin, et al., "Bragg gratings inscribed in selectively inflated photonic crystal fibers," Optics Express, 2017, 25(23): 28442-28450.

[8] C. Wang, J. C. Zhang, C. Z. Zhang, J. He, Y. C. Lin, W. Jin, et al., "Bragg gratings in suspended-core photonic microcells for high-temperature applications," Journal of Lightwave Technology,
2018, 36(14): 2920-2924.

[9] C. Liao, D. Wang, Y. Li, T. Sun, and K. T. V. Grattan, "Temporal thermal response of type II-IR fiber Bragg gratings," Applied Optics, 2009, 48(16): 3001-3007.

[10] G. Brambilla, V. Finazzi, and D. J. Richardson, "Ultra-low-loss optical fiber nanotapers," Optics Express, 2004, 12(10): 2258-2263.

[11] L. M. Tong, R. R. Gattass, J. B. Ashcom, S. He, J. Lou, M. Shen, et al., "Subwavelength-diameter silica wires for low-loss optical wave guiding," Nature, 2003, 426(6968): 816-819.

[12] C. Liao, Q. Wang, L. Xu, S. Liu, J. He, J. Zhao, et al., "D-shaped fiber grating refractive index sensor induced by an ultrashort pulse laser," Applied Optics, 2016, 55(7): 1525-1529.

[13] C. Lin, C. Liao, J. Wang, J. He, W. Ying, Z. Y. Li, et al., "Fiber surface Bragg grating waveguide for refractive index measurements," Optics Letters, 2017, 42(9): 1684-1687.

[14] X. Fang, C. Liao, and D. Wang, "Femtosecond laser fabricated fiber Bragg grating in microfiber for refractive index sensing," Optics Letters, 2010, 35(7): 1007-1009.

[15] T. Geernaert, K. Kalli, C. Koutsides, M. Komodromos, T. Nasilowski, W. Urbanczyk, et al., "Point-by-point fiber Bragg grating inscription in free-standing step-index and photonic crystal fibers using near-IR femtosecond laser," Optics Letters, 2010, 35(10): 1647-1649.

[16] Y. Zhang, B. Lin, S. C. Tjin, H. Zhang, G. Wang, P. Shum, et al., "Refractive index sensing based on higher-order mode reflection of a microfiber Bragg grating," Optics Express, 2010, 18(25): 26345-26350.

[17] Y. Ran, Y. Tan, L. Sun, S. Gao, J. Li, L. Jin, et al., "193nm excimer laser inscribed Bragg gratings in microfibers for refractive index sensing," Optics Express, 2011, 19(19): 18577-18583.

[18] A. J. C. Grellier, N. K. Zayer, and C. N. Pannell, "Heat transfer modelling in $\mathrm{CO}_{2}$ laser processing of optical fibres," Optics Communications, 1998, 152(4-6): 324-328.

[19] M. Sumetsky, Y. Dulashko, J. M. Fini, A. Hale, and D. J. Digiovanni, "The microfiber loop resonator: theory, experiment, and application," Journal of Lightwave Technology, 2006, 24(1): 242-250.

[20] R. W. Lewis, P. Nithiarasu, and K. N. Seetharamu, Fundamentals of the finite element methods for heat and fluid flow. England: John Wiley \& Sons Ltd., 2004. 